\title{
Pionierbereich: Ambulante Kinder- und Jugendrehabilitation
}

\author{
Den Behandlungserfolg und die Teilhabe sichern Nicht für alle Kinder und Ju- \\ gendliche mit chronischen Erkrankungen und deren Familien kommt eine statio- \\ näre Rehabilitation in Frage. Durch eine Gesetzesänderung können nun auch \\ ambulante Reha-Angebote für Kinder und Jugendliche jedes Jahr wahrgenom- \\ men werden. Eines der (noch) raren Angebote gibt es an der UniReha in Köln. Ein \\ Praxisbericht. " Christina Stark, Miriam Jackels, Evelyn Alberg, Eckhard Schönau
}

\section{ZUSAMMENFASSUNG}

Seit der Änderung des Flexirentengesetzes 2017 kann die medizinische Rehabilitation von Kindern und Jugendlichen auch als ambulante Leistung jährlich erbracht werden. Die Angebote für ambulante Kinder- und Jugendrehabilitation sind im Aufbau, jedoch bieten einige Standorte bereits ambulante Rehabilitation für Kinder- und Jugendliche an. Zu diesen gehört die UniReha GmbH, Zentrum für Prävention und Rehabilitation der Uniklinik Köln. Ein zentrales Anliegen der ambulanten Rehabilitation ist die Reha-Leistung näher an das Umfeld der Kinder und Jugendlichen zu rücken. Neben dem Praxisbericht der Kölner ambulanten Kinder- und Jugendrehabilitation werden nützliche Tipps für die Beratung von Patienten und ihren Angehörigen gegeben.

\section{Schlüsselwörter: ambulante Kinderrehabilitation, Kinder und Jugendliche, Funktionsstörungen, Teilhabe, Medizinische Rehabilitation, Rehakette}

aut den Zahlen der Kinder- und Jugendgesundheitsstudie (KiGGS) leiden ca. 4,3\% aller Kinder und Jugendlichen in Deutschland (ca. 500.000) an gesundheitlichen Einschränkungen. Chronische Krankheiten gehen sehr häufig mit anhaltenden Funktions- und Teilhabestörungen einher, die zu Beeinträchtigungen und Benachteiligungen in allen Lebensbereichen führen können. Chronische Erkrankungen des Kindes- und Jugendalters mit anhaltenden Funktionsstörungen können lebenslang bestehen und gefährden die gesunde körperliche und geistige Entwicklung. Die Leistungsfähigkeit und Teilhabe des späteren Erwerbslebens ist bedroht. Die medizinische Rehabilitation ist unter medizini- schen Gesichtspunkten ein wichtiger Bestandteil des Gesamtbehandlungskonzeptes, um nach akuten und chronischen Erkrankungen den erzielten Behandlungserfolg und den weiteren Genesungsprozess zu sichern, Funktions- und Teilhabestörungen zu verringern oder zu beseitigen und Benachteiligungen zu vermeiden sowie eine altersgerechte Entwicklung und Teilhabe zu fördern. Daher sollte die Initiierung und Durchführung einer Rehabilitationsbehandlung frühzeitig in Erwägung gezogen werden.

Seit der Änderung des \$15a SGB VI (Flexirentengesetz) im Jahr 2017 ist die bis dahin bestehende Beschränkung auf stationäre Leistungen für die medizinische Rehabilitation von Kindern und Jugendlichen entfallen und diese kann jetzt auch als ambulante Leistung erbracht werden. Zudem ist sie nun keine Ermessensleistung mehr, sondern wurde in den Pflichtkatalog der Deutschen Rentenversicherung (DRV) aufgenommen und kann jedes Jahr wiederholt werden.

\section{Vorteile einer ambulanten Rehabilitation}

Laut den „Allgemeinen Rahmenempfehlungen zur ambulanten Rehabilitation von Kindern und Jugendlichen“ der Deutschen Gesellschaft für Sozialpädiatrie und Jugendmedizin und der Fachgesellschaft für Rehabilitation im Kindes und Jugendalter unterscheiden sich die Rehabilitationsziele der ambulanten und stationären Rehabilitation nicht. Eine ambulante Rehabilitation sei jedoch vorzuziehen, wenn deren Ziele auch ambulant erreicht werden könnten. Zudem kommt für einige Familien eine stationäre Rehabilitation nicht in Frage. Gründe hierfür können sein, dass es sich die Eltern beispielsweise aus beruflichen Gründen nicht leisten können, das Kind auf einen mehrwöchigen stationären Reha-Aufenthalt zu begleiten. Oder dass die Schulversäumnisse trotz Überbrückungsunterricht in der stationären Rehabilitation als zu relevant eingeschätzt werden.

Die Stärken einer ambulanten Rehabilitation für Kinder und Jugendliche sind: 
- Verstärkte Einbeziehung der Familien (Selbstmanagement „Empowerment“)

- Zeitliche Verknüpfung mit dem Schulbesuch bzw. von Ausbildungsverpflichtungen

_ Durchführung einer Rehabilitationsmaßnahme über einen längeren Zeitraum zur Steigerung von Lern- und Trainingseffekten

- Verknüpfung mit gezielten rehabilitativen Maßnahmen im Kindergarten, Schule oder am Arbeitsplatz in der Alltagssituation

_ Wohnortnahes interdisziplinäres therapeutisches Angebot

Abzuwägen bei der Indikationsstellung ambulant versus stationär sind laut den Rahmenempfehlungen insbesondere die folgenden Aspekte:

- Ist eine vorübergehende Herausnahme aus dem sozialen Kontext erforderlich und sinnvoll, um das Rehabilitationsziel zu erreichen?

- Welcher Zeitrahmen und welche Intensität der Rehabilitationsangebote sind geeignet, die Rehabilitationsziele zu erreichen?

- Welche Möglichkeiten der direkten Einflussnahme auf Kontextfaktoren und deren Veränderungspotenzial (Familie und weiteres soziales Umfeld) bestehen?

- Wie kann die Kontinuität der ärztlichen und therapeutischen Bezugspersonen in der Rehabilitation und Nachsorge bzw. Nachhaltigkeit der therapeutischen Erfolge, Transfer der erlernten Methoden in das heimatnahe Umfeld („setting“) gesichert werden (Rehakette)?

\section{Ambulante Angebote im Aufbau}

Es mangelt bisher bundesweit an entsprechenden Angeboten der ambulanten Kinder- und Jugendrehabilitation, jedoch sind einige im Aufbau, und es lohnt sich, hier die schnelle Entwicklung neuer Angebote zu verfolgen, beispielsweise über die Homepage des Bündnisses für Kinder- und Jugendrehabilitation (Kasten). Einige Standorte bieten bereits ambulante Rehabilitation für Kinder- und Jugendliche an. Zu diesen gehört die UniReha $\mathrm{GmbH}$, Zentrum für Prävention und Rehabilitation der Uniklinik Köln. Hier sind Angebote der Kinder- und Jugendrehabilitation nichts Neues, denn das Unternehmen ist seit vielen Jahren auf Kinder- und Jugendrehabilitation spezialisiert. Seit 2019 wird hier nun die ambulante Medizinisch-Lebenswelt orientierte Rehabilitation (AMLOR) für Kinder und Jugendliche angeboten. Gestartet wurden die Idikationen neuromuskuläre Erkrankungen, Adipositas und skelettale Erkrankungen.

Ein zentrales Anliegen der ambulanten Form ist, die Reha-Leistung näher an das Umfeld der Kinder und Jugendlichen zu rücken, beziehungsweise die Familien in ihrem häuslichen Umfeld zu beraten. Das Kölner AMLOR-Konzept begleitet das Kind/den Jugendlichen und seine Familie über ein halbes Jahr. Ein Schwerpunkt liegt auf dem individuellen, intensiven Heimtrainingsprogramm, wozu die Kinder/Jugendlichen und deren Eltern in drei IntensivWochen angeleitet werden. Somit werden bei minimalem Zeitaufwand und höchster Effektivität der Schulbesuch oder die Ausbildung nur wenig beeinträchtigt.

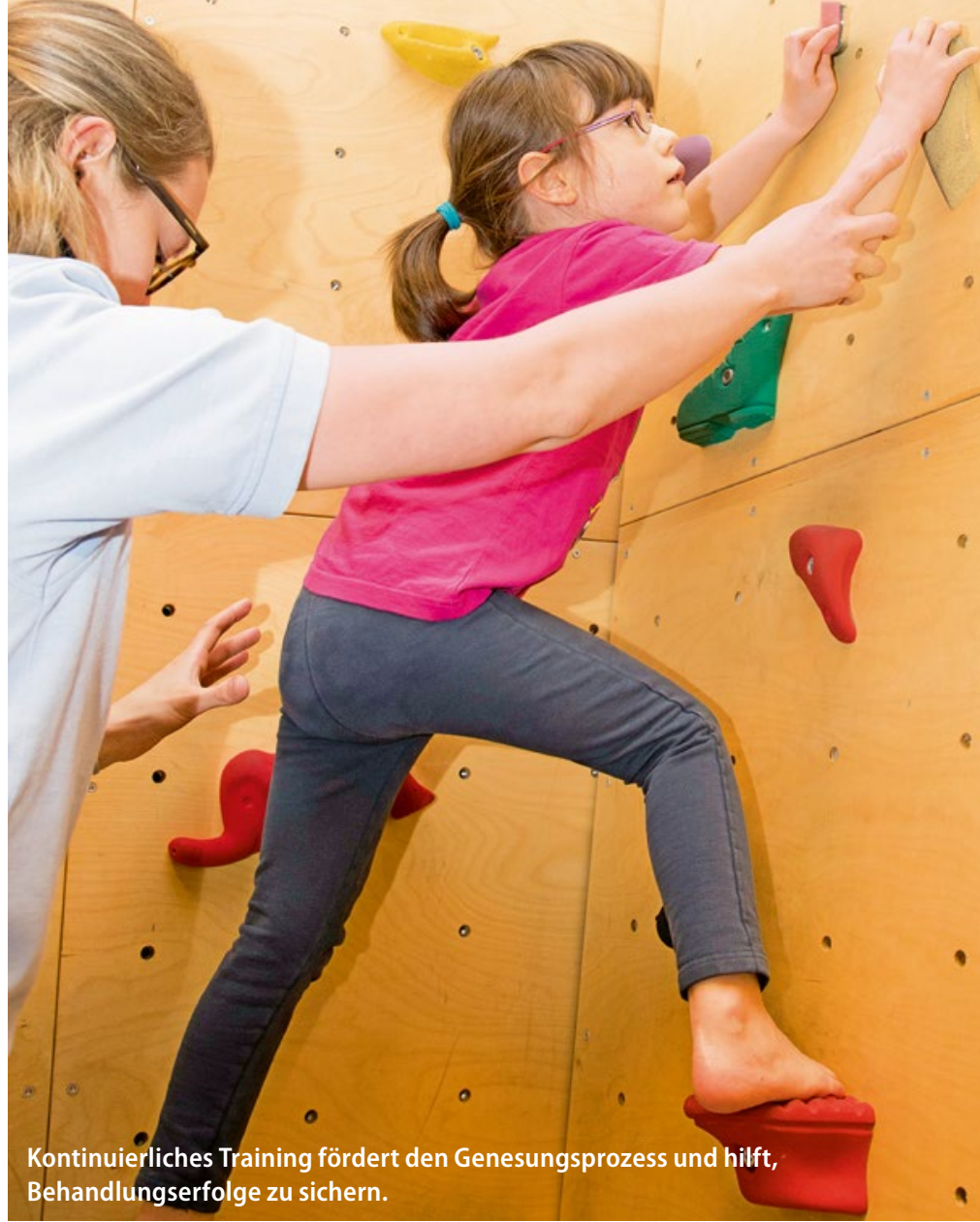

\section{AMLOR: Medizinisch-lebenswelt orientiertes Konzept mit dem Ziel, Nähe zu schaffen}

Die ambulante Rehabilitation für Kinder und Jugendliche (AMLOR) in Köln startet mit einer Intensiv-Woche, in der therapeutische Bewegungsangebote mit Schulung und Beratung kombiniert werden (Abb. 1). Der familiäre Alltag soll dabei weitestgehend aufrechterhalten werden. Die Intensiv-Woche findet also am Nachmittag statt; in der Regel werden sie nach zwei und vier Monaten wiederholt. Die Inhalte passen sich dem Trainingsfortschritt an. Zwischen den Intensiv-Wochen findet meist alle zwei Wochen ein Präsenznachmittag statt. Für Kinder und Jugendliche mit Adipositas finden insgesamt zwölf Präsenztermine statt, wobei diese (im Vergleich zu den anderen Modulen) in den ersten zwei Monaten wöchentlich terminiert werden. Hier wiederholen sich in jeweils drei Einheiten die Inhalte der Intensiv-Wochen (Bewegung und Schulung) indikationsspezifisch, und das Heimtraining wird kontinuierlich begleitet.

Zwei Präsenztage werden im individuellen häuslichen Umfeld abgehalten - zu Hause, in der Schule oder im Kindergarten. Diese Termine sind sehr wichtig, um die Rehabilitationsziele und das häusliche Training konkret dort umzusetzen, wo der Alltag stattfindet. Dies ist von elementarer Bedeutung, da häufig die in der Rehabilitationseinrichtung erreichten Ziele im Anschluss im Alltag untergehen. Beispielsweise bei Adipositas werden auch Exkursionen zu lokalen Sportvereinen angeboten, um die Überleitung in lokale Vereine und/oder Selbsthilfegruppen optimal zu gestalten. Dies hat sich als sehr wertvoll herausgestellt: So konnte die zuletzt abgeschlossene Gruppe des Moduls Adipositas der AMLOR in Köln ausnahmslos in einen lokalen Sportverein übergeleitet werden. Solche Erfolge motivieren für das Weiterführen und einen Ausbau der ambulanten Rehabilitationsangebote. 
Abb. 1: Ablauf der ambulanten Medizinisch-Lebenswelt orientierten Rehabilitation (AMLOR) für Kinder und Jugendliche der UniReha GmbH, Zentrum für Prävention und Rehabilitation der Uniklinik Köln

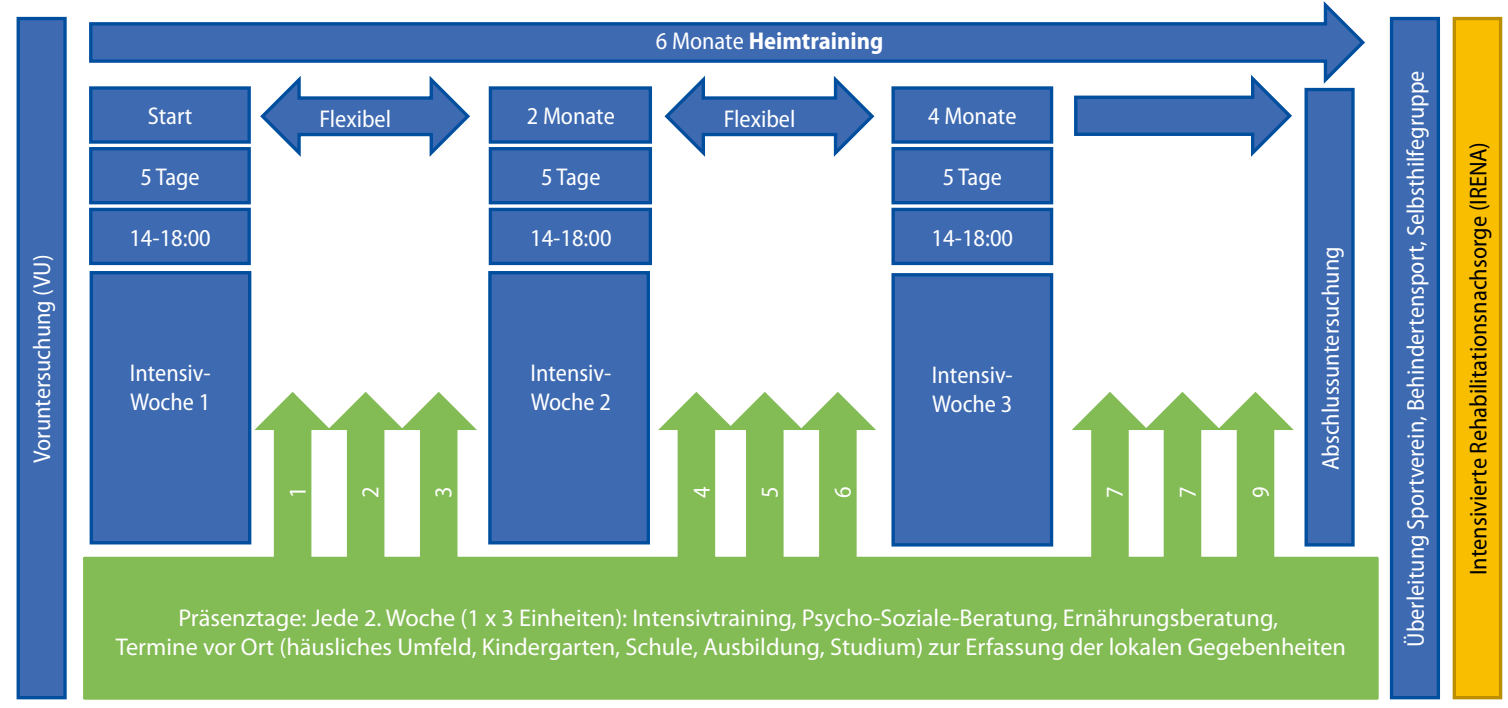

\section{CHECKLISTE: ANTRAG AUF REHABILITATION}

\section{Wann kann ein Reha-Antrag gestellt werden?}

$\checkmark$ Generell kann bei chronischen, somatischen oder psychischen Erkrankungen, nach akuten Erkrankungen oder nach Unfallverletzungen, mit jeweils anhaltender Funktions- und Teilhabestörung ein Antrag auf Kinder- und Jugendrehabilitation beim zuständigen Rentenversicherungsträger gestellt werden.

$\checkmark$ Für eine ambulante Kinder-und Jugendrehabilitation ist zu prüfen, wo und ob diese Form im entsprechenden Einzugsgebiet angeboten wird.

\section{Wer kann einen Antrag stellen?}

$\checkmark$ Leistungen zur medizinischen Rehabilitation gehören zu den sogenannten Antragsleistungen. Erziehungsberechtigte können den Antrag beim zuständigen Rentenversicherungsträger stellen, wenn die versicherungsrechtlichen und persönlichen Voraussetzungen gegeben sind.

$\checkmark$ Sind die Eltern unterschiedlich rentenversichert, können sie auswählen, bei welchem Rentenversicherungsträger sie den Antrag stellen.

$\checkmark$ Der behandelnde Kinder- und Jugendarzt, Hausarzt oder Kinder- und Jugendpsychiater unterstützt den Antrag mit einem ärztlichen Befundbericht.

Was wird für den Antrag benötigt?

$\checkmark$ Antrag auf Leistungen zur Rehabilitation für Kinder und Jugendliche (Kinderrehabilitation) (G0200, von den Eltern auszufüllen). $\checkmark$ Ärztlicher Befundbericht zum Antrag auf Leistungen zur Rehabilitation von Kindern und Jugendlichen (Kinderrehabilitation) (G0612) mit Honorarabrechnung (G0600).

$\checkmark$ Formulare und Informationen: deutsche-rentenversicherung.de

\section{Wo wird der Antrag gestellt?}

$\checkmark$ Der Antrag wird beim zuständigen Rentenversicherungsträger gestellt. Auf der jeweiligen jährlichen Renteninformation steht, welche Rentenversicherung zuständig ist.

$\checkmark$ Die Antragsunterlagen sind postalisch einzureichen.

$\checkmark$ Achtung: Die Rentenversicherung prüft die medizinischen und persönlichen Voraussetzungen. Da die ambulanten Reha-Angebote für Kinder und Jugendliche im Aufbau sind, gibt es noch keine Formulare, die den Anforderungen der ambulanten Leistung angepasst sind. Bei Problemen ist der persönliche Kontakt zum Sachbearbeiter zu suchen.

Weitere Informationen zur Kinder- und Jugendrehabilitation: Bündnis Kinder- und Jugendreha im Netz e.V. (BKJR)

kinder-und-jugendreha-im-netz.de

$\Delta$ „Handbuch Medizinische Rehabilitation für Kinder und Jugendliche" 


\section{Intensive Reha-Nachsorge: Anschluss und Überleitung}

Besonders wichtig ist die Überleitung der Rehabilitationsergebnisse in den Alltag und das häusliche Umfeld, in ortsnahe Sportvereine, Behindertensport- und/oder Selbsthilfegruppen für eine nachhaltige Verhaltensänderung und kontinuierliches Training zum Erhalt bzw. der weiteren Verbesserung der Fähigkeiten. Im Anschluss kann auch eine Überleitung in die Intensive Rehabilitationsnachsorge (IRENA) erfolgen (Abb. 1). Die IRENA ist ebenfalls eine Leistung der Deutschen Rentenversicherung und kann direkt im Anschluss an die stationäre oder, wie in diesem Fall, ambulante Rehabilitation beantragt werden. Die UniReha in Köln bietet sie für Kinder und Jugendliche an.

\section{Tipps für die Praxis und die Rolle der Pflege}

Im Rahmen der ambulanten Rehabilitationsangebote für Kinder und Jugendliche kommt der Pflege die Rolle der aufmerksamen Beobachtung der Entwicklung in diesem Sektor der Gesundheitsversorgung und Aufklärung der Patienten und deren Familien zu. Sollte ein Kind oder Jugendlicher für ein ambulantes Rehabilitationsangebot in Frage kommen, sind verschiedene Fragen zu beantworten, die das Antragsverfahren betreffen (Checkbox). Die hier in Kurzform dargestellten Informationen werden ausführlich auch im Handbuch Medizinische Rehabilitation für Kinder und Jugendliche beschrieben, das von der Deutschen Rentenversicherung Bund in Zusammenarbeit mit dem Bündnis Kinder- und Jugendreha und dem Berufsverband der Kinder- und Jugendärzte herausgegeben wird. \II

Hier steht eine Anzeige.

\section{FAZIT}

Seit Änderung des Flexirentengesetzes kann die medizinische Rehabilitation von Kindern und Jugendlichen auch als ambulante Leistung jährlich erbracht werden.

Die Angebote für ambulante Kinder- und Jugendrehabilitation sind im Aufbau.

Die bereits angebotenen Strukturen, z.B. von der UniReha $\mathrm{GmbH}$, Zentrum für Prävention und Rehabilitation der Uniklinik Köln, werden gut angenommen.

\section{Autorenkontakt:}

Dr. rer. medic. Christina Stark, Physiotherapeutin und verantwortlich für Forschung und Entwicklung der UniReha GmbH, Zentrum für Prävention und Rehabilitation der Uniklinik Köln; wissenschaftliche Mitarbeiterin der Arbeitsgruppe Prof. Eckhard Schönau an der Klinik und Poliklinik für Kinderund Jugendmedizin der Uniklinik Köln.

E-Mail: christina.stark@uk-koeln.de

Ko-Autoren: Dr. med. Miriam Jackels, Fachärztin für Kinder- und Jugendmedizin mit Schwerpunkt pädiatrische Endokrinologie und Diabetologie und Adipositastrainerin; Dr. med. Evelyn Alberg, Fachärztin für Kinder- und Jugendmedizin mit Schwerpunkt Neuropädiatrie; Prof. Dr. med. Eckhard Schönau, Facharzt für Kinder- und Jugendmedizin und Oberarzt an der Klinik und Poliklinik für Kinder- und Jugendmedizin der Uniklinik Köln; ärztlicher Leiter und Geschäftsführer der UniReha GmbH 